\title{
Téoros
}

Revue de recherche en tourisme

\section{Entre local et régional : le foncier dans les politiques de financement du développement touristique en Grèce}

\section{Nikolaos Triantafyllopoulos}

Volume 27, numéro 2, été 2008

URI : https://id.erudit.org/iderudit/1070797ar

DOI : https://doi.org/10.7202/1070797ar

Aller au sommaire du numéro

Éditeur(s)

Université du Québec à Montréal

ISSN

0712-8657 (imprimé)

1923-2705 (numérique)

Découvrir la revue

Citer cet article

Triantafyllopoulos, N. (2008). Entre local et régional : le foncier dans les politiques de financement du développement touristique en Grèce. Téoros, 27(2), 49-58. https://doi.org/10.7202/1070797ar d'utilisation que vous pouvez consulter en ligne. 


\section{Entre local et régional : le foncier dans les politiques de financement du développement touristique en Grèce}

\section{Nikolaos Triantafyllopoulos}

Jusqu'à présent, une politique rigoureuse de développement touristique, dans le sens de l'élaboration de stratégies cohérentes et de coordonnées traitant du tourisme dans sa globalité, suivie d'actions concertées en vue de l'obtention de résultats concrets et à long terme, n'a jamais existé en Grèce. Le ministère chargé de l'Aménagement et de l'Environnement a indirectement influencé le développement spatial du tourisme, soit par le biais de politiques d'aménagement et d'urbanisme, soit par d'autres mesures s'y rapportant. Les instances étatiques vouées exclusivement au tourisme, l'Office hellénique du tourisme et le ministère du Tourisme (lorsqu'il existe), n'ont exercé que des actions directes, plutôt ponctuelles, qui n'ont pas toujours eu des effets importants sur le territoire. Quant au ministère de l'Aménagement et de l'Environnement, il n'a jamais élaboré ni institué de plan de développement du tourisme à l'échelle nationale ou régionale. C'est le ministère de l'Économie nationale qui a mené des actions importantes en matière de tourisme, grâce principalement à des politiques de développement régional, matérialisées par les lois de développement régional successives et des politiques de financement du secteur par des banques. Au cours des quarante dernières années, l'évolution de la capacité hôtelière a été spectaculaire dans certains départements et régions du pays (ill. 1), avec de fortes concentrations à l'échelle locale. Quelles que soient les modalités des aides au développement, ces politiques ont eu des effets discutables : elles ont contribué au développement de l'offre en hébergements, mais elles n'ont pas empêché le développement inégal des régions ayant des prédispositions en matière de

\section{Illustration 1}

Évolution de la capacité hôtelière en Grèce entre 1971 et 2005

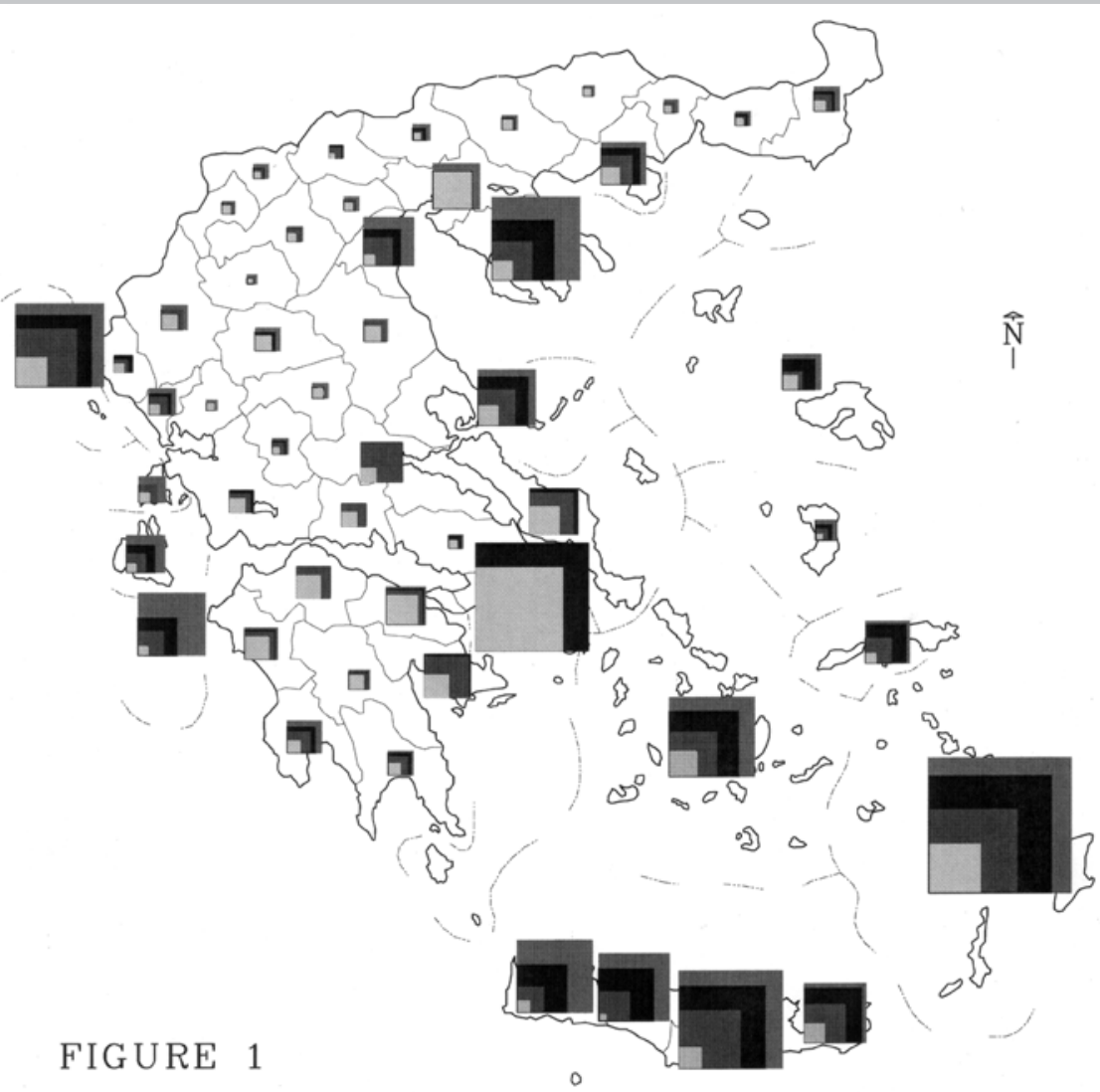

GRECE: EVOLUTION DE LA CAPACITE HOTELIERE 1971-1981-1993-2005
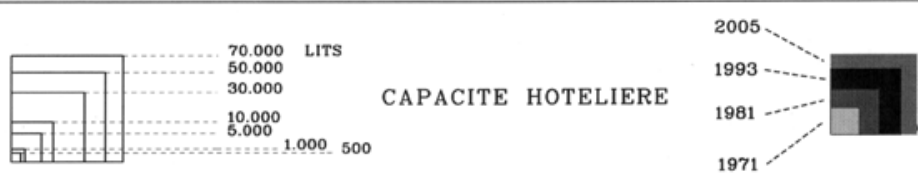

REALISATION: N. TRIANTAFYLLOPOULOS

SOURCE DES DONNEES : OFFICE NATIONAL DE STATISTIQUES 
tourisme, et elles ont entraîné l'apparition de problèmes spatiaux et économiques à l'échelle locale. L'urbanisation touristique en Grèce prend localement, le plus souvent, la forme d'un déploiement diffus, anarchique et linéaire des équipements le long des côtes, ce qui conduit à la consommation immodérée et à la dégradation des espaces littoraux fragiles (Triantafyllopoulos, 1999).

Les politiques de développement régional du tourisme exercées par le ministère de l'Économie revêtent une importance prépondérante, car la presque totalité des investissements hôteliers ont été réalisés avec l'aide de l'État. Parallèlement aux aides et aux subventions en faveur du tourisme, le système bancaire, contrôlé par l'État jusqu'à la fin des années 1990, a assuré l'approvisionnement du secteur en capitaux abondants.

L'analyse de la bibliographie internationale sur le développement du tourisme fait ressortir une absence frappante d'études ou même de références aux politiques de financement du secteur en faveur de son développement régional (Cazes, 1992 ; Seaton et al., 1994 ; Bar-On et Even-Zahev, 1995 ; Montanari et Williams, 1995 ; Igoumenakis, 1997 ; loannides et Debagge, 1997 ; Cooper et al., 1998 ; Pearce et Butler, 1999 ; Weaver, 2001), à l'exception d'une courte référence sur Chypre (WTO, 1994). Dans les articles relatifs à cette question, les références sont plutôt vagues (Sinclair et Page, 1993 ; Göymen, 2000 ; Ivars-Baidal, 2004). De même, les références concernant le tourisme sont presque inexistantes dans les manuels sur les politiques de développement régional (Artobolevskiy, 1997 ; Armstrong et Taylor, 2000 ; Capello, 2007 et, pour la Grèce, Papadoulis et al., 2004 ; Petrakos et Psycharis, 2004), à quelques très rares exceptions, nettement insuffisantes (dont Vanhove, 1999). Les chercheurs se sont régulièrement intéressés à l'industrie ou à d'autres sujets transversaux comme la recherche et développement (R\&D), I'innovation, les petites et moyennes entreprises, mais le tourisme n'entre presque jamais dans leurs problématiques. Les particularités du fait touristique et de sa dépendance vis-à-vis des constantes foncières n'y sont pas considérées. En plus, les chercheurs semblent se préoccuper presque exclusivement des effets globaux des politiques exercées. Plus précisément, ils accordent de l'importance aux fonctions des divers outils destinés à promouvoir le développement régional, mais quasi uniquement quand leurs résultats sont quantifiables et qu'ils permettent un traitement statistique (Wren, 2005). Par exemple, les effets et les fonctions " secondaires ", comme le rôle du foncier dans la mise en œuvre des politiques, ne sont, à notre connaissance, jamais pris en considération. Difficilement quantifiable, tant en termes de surfaces qu'en termes de valeur, le foncier a complètement échappé à la recherche.

L'espace en tant que support du tourisme impose sa " consommation sur place " des services touristiques. Le foncier est une matière première du développement touristique, en termes spatiaux comme financiers. Hétérogène et infongible, le foncier acquiert dans les espaces touristiques une valeur autant symbolique que réelle ou même vénale. II entre donc dans le processus de production touristique en tant que valeur économique et financière. L'hôtellerie possède des caractéristiques qui lui confèrent des dimensions d'industrie lourde : coûts élevés des équipements, forte intensité capitalistique et amortissement lent des investissements. À titre de produit touristique de base ordinaire et localisé, elle occupe une place dans l'analyse du rôle du foncier dans la mise en œuvre des politiques de financement du tourisme en faveur de son développement régional. L'objectif de ce travail n'est pas d'examiner la myriade des facteurs qui, en relation avec le foncier, produisent le développement touristique sur les plans régional et local. II examine plutôt les fonctions de la constante foncière dans l'outil le plus important de politique régionale en matière de tourisme : les lois de développement régional, depuis les années 1950 jusqu'au début du nouveau siècle ; il retrace aussi quelques effets engendrés sur le développement des espaces locaux, là où se matérialisent leurs dispositifs de la manière la plus concrète. Enfin, cette étude propose une méthode d'appréhension des effets des incitations en faveur du développement régional du tourisme, au moyen d'une technique commune d'évaluation des investissements immobiliers touristiques, fondée sur la théorie de la rente foncière et sur celle des effets externes, telles qu'elles sont développées dans les travaux d'Alfred Marshall (1890).

\section{Les aides directes de l'État au développement régional du tourisme}

Selon les dispositifs des lois de développement régional, un investissement touristique comprend trois composantes majeures : a) les aides de l'État qui prennent la forme de subventions aux entreprises, de subventions portant sur les taux d'intérêt des prêts bancaires, d'exonérations fiscales et d'autres incitations diverses (création d'emplois, leasing pour l'acquisition d'équipement), b) les prêts bancaires et c) les capitaux propres de l'investisseur. Les diverses incitations accordées par les lois sur la création d'entreprises hôtelières visent soit à diminuer l'importance des capitaux propres et le coût des capitaux empruntés, soit des exonérations fiscales.

\section{Les années 1950 et $1960:$ le tourisme attire l'attention de l'État}

Au début des années 1950, le pays sort très traumatisé d'une période de guerres et de catastrophes successives qui ont duré plus de un siècle ; la Deuxième Guerre mondiale et l'atroce guerre civile qui l'a suivie ne s'achèvent qu'en 1949. L'exode rural et l'immigration s'accentuent et le processus de développement excessif d'Athènes est déjà entamé. Le gouvernement se rend compte du développement inégal du pays et il institue pour la première fois dans les années 1950 (décrets de Loi 2176/1952 et 2687/1953) des aides en faveur du développement régional du pays, dans le but d'attirer des investissements étrangers et de favoriser l'importation de capitaux étrangers. Le tourisme gagne l'attention des instances gouvernementales qui commencent, mutatis mutandis, à se préoccuper de son développement, bien qu'à l'époque il ne soit encore qu'une activité marginale.

Ce n'est que dans le plan quinquennal 19601964 que le tourisme est considéré comme un secteur de développement prioritaire. Le principal objectif de l'État est le développement rapide du secteur, plus particulièrement l'augmentation de l'offre en hébergements et des recettes touristiques. Les incitations adoptées en faveur du tourisme par la Loi 4171/1961 sont mineures, principalement fiscales. C'est la Loi 543/1968 qui assure pour la première fois des aides spécifiques 
au secteur touristique ; à noter que la contribution minimale de l'entrepreneur à la création d'une entreprise hôtelière est alors fixée à $20 \%$, y compris la valeur du terrain, le reste étant subventionné par l'État.

\section{Les années 1970 : incertitudes et réticences}

En 1972, pour la première fois, le pays est divisé en trois zones d'incitation au développement touristique (décret de Loi 1313/1972) (ill. 2) et c'est en fonction de ces zones (la zone A est la moins favorisée) que les aides au développement régional sont allouées. La période d'application de ce système d'aide est caractérisée par une instabilité politique et économique. Dans les années 1970, la première crise pétrolière engendre des déséquilibres économiques importants : I'inflation explose et pèse considérablement sur le coût de fonctionnement des entreprises. Les hôteliers, s'appuyant sur les aides de l'État, maintiennent les prix à des niveaux bas (CPRE, 1976).
Après la chute du régime dictatorial des colonels et l'invasion turque à Chypre en 1974 les menaces de guerre provoquent une réduction importante des arrivées touristiques. Les lois 289/1976 et 849/1978 visent à susciter un intérêt pour l'investissement dans les régions frontalières et à remonter le moral des habitants des îles égéennes. Une nouvelle zone d'incitation est créée par cette loi, qui comprend - outre les départements du nord du pays - toutes les îles voisines de la Turquie, menacées de dépeuplement. Parmi les mesures adoptées, citons le taux de subvention des entreprises relevé jusqu'à $85 \%$ et la participation minimale de l'entrepreneur ramenée à $15 \%$.

\section{Les années 1980 : la « belle époque " des subventions}

Devant l'incapacité des politiques jusque-là adoptées à promouvoir un développement régional du tourisme au niveau souhaité, l'État institue un nouveau système d'aides au secteur. Aux termes de la Loi 1116/1981, les subventions en capitaux pour la création d'entreprises peuvent atteindre $80 \%$ du coût de l'investissement. L'achat de terrains urbains n'est pas subventionné, contrairement aux terrains non urbains. Étant donné l'absence de plans d'aménagement et d'urbanisme, cette loi favorise l'expansion des équipements touristiques dans l'espace rural, c'est-à-dire en dehors des plans d'urbanisme.

Les lois relatives au développement constituent un des moyens de mise en œuvre des politiques économiques, car, grâce à ces lois, le gouvernement exerce son contrôle non seulement sur le développement, mais aussi sur les moyens de production. C'est ainsi qu'après l'arrivée au pouvoir des socialistes, en 1981, la nouvelle Loi 1262/1982 impose une participation de l'État au fonds social des entreprises à plus de 300 lits, qui sont subventionnées en capitaux; en outre, l'État subventionne les taux d'intérêt des prêts bancaires des entreprises (jusqu'à hauteur

\section{Illustration 2}

\section{Les zones d'incitation au développement touristique selon le décret de loi 1313/1972}

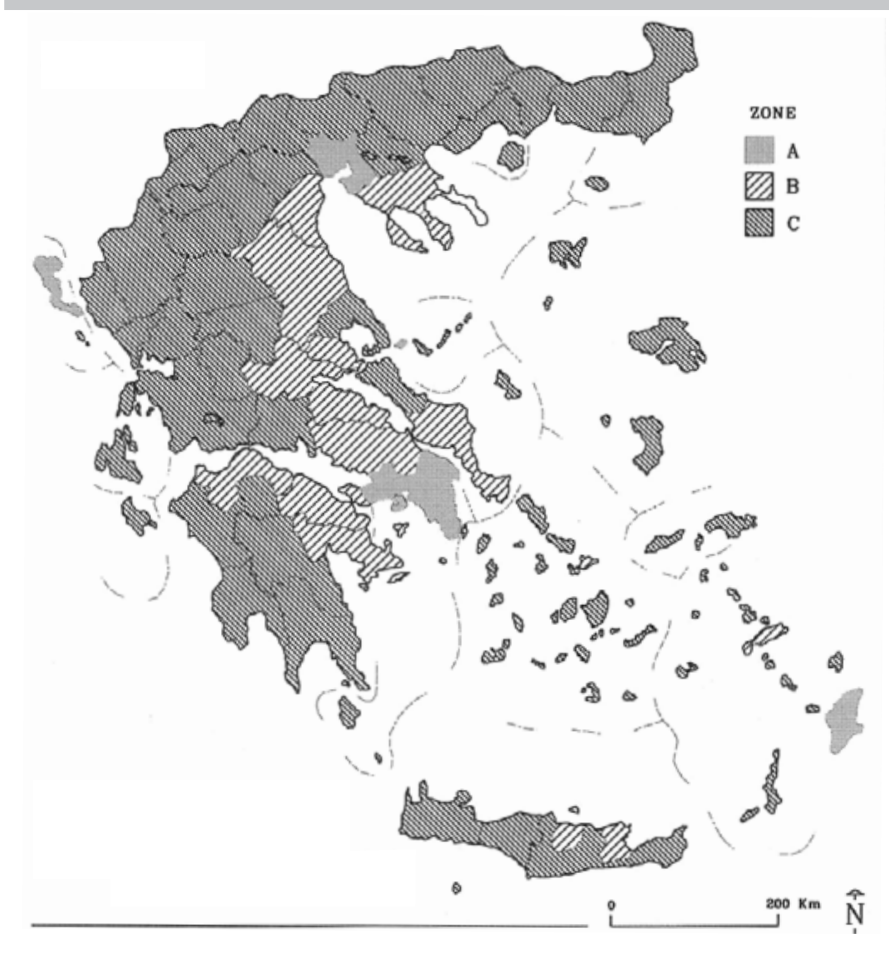

Source : auteur.

\section{Illustration 3}

\section{Les zones d'incitation au développement touristique selon les lois $1262 / 82$ et $1892 / 90$}

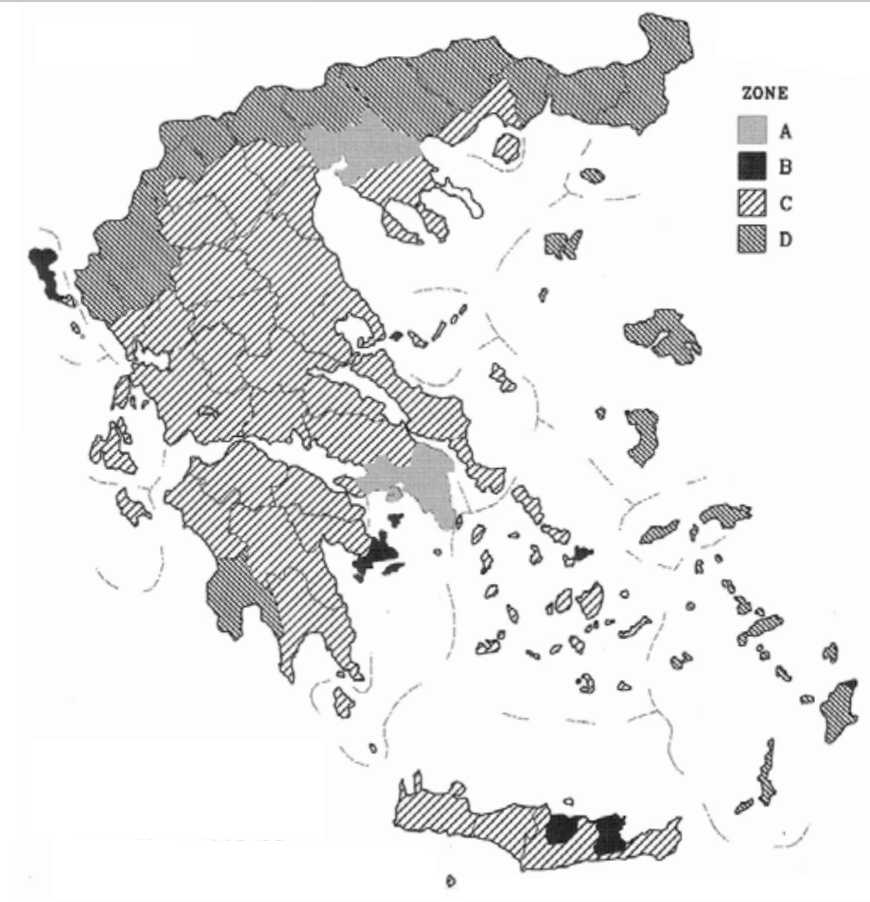

Source : auteur. 
de 50 \%). Le pays est divisé en quatre zones d'incitation spécifiques au tourisme. L'achat du terrain n'est pas subventionné, mais sa valeur est comprise dans le montant de la participation de l'entrepreneur au coût de la réalisation. À partir de 1986 et jusqu'en 1993, la création (l'extension ou la rénovation) d'entreprises touristiques est financée par les fonds des "Programmes globaux méditerranéens " de la Communauté économique européenne. Ce type de financement est plus avantageux, car il assure des subventions de 10 à $20 \%$ en sus de celles prévues par la Loi 1262/1982. Prêts bancaires à part, l'entrepreneur doit contribuer de 15 à $30 \%$ du coût total de l'investissement, y compris la valeur du terrain.

\section{Vers le nouveau siècle : un avenir incertain ou prometteur?}

Quelques mois après l'ascension au pouvoir de la droite, une nouvelle loi relative au développement est votée (Loi 1892/1990). Le gouvernement fait part de son objectif de contrôler l'offre touristique au profit de la qualité d'accueil. Parmi les buts de cette politique figurent : la construction d'infrastructures et d'hôtels de catégorie supérieure, l'encouragement aux nouvelles formes de tourisme (villages vacances, tourisme vert, tourisme en montagne, stations de ski, thermalisme), la création de nouveaux casinos, l'encouragement à la reconversion des bâtiments traditionnels en établissements touristiques et l'utilisation plus rationnelle du sol. Les quatre zones d'incitation de la loi précédente sont en principe maintenues (ill. 3). Le taux de subvention oscille de $10 \%$ à $25 \%$ du montant total de l'investissement touristique productif. Les taux d'intérêt des prêts bancaires sont aussi subventionnés au même pourcentage que les subventions accordées. Les entrepreneurs doivent participer à hauteur de 30-40 \% du coût de l'investissement. II faut signaler que l'achat du terrain n'est pas subventionné et que, pour la première fois, sa valeur n'est pas prise en compte dans le montant de l'investissement subventionné.

En 1998 est votée la nouvelle Loi 2601/1998, relative au développement régional. Le territoire national y est toujours divisé en quatre zones ; l'innovation de cette loi réside dans l'introduction de critères autres que spatiaux pour la délimitation des zones, comme le déclin de la population active ou le taux de chômage par rapport à la moyenne nationale (ce qui ne permet pas une représentation cartographique de ces zones). Pourtant, il est à noter que, paradoxalement, des régions excessivement développées en matière de tourisme sont parmi les plus favorisées. Les subventions atteignent $25 \%$ du montant de l'investissement hôtelier, tandis que les capitaux propres ne doivent pas être inférieurs à $40 \%$ de l'investissement, y compris la valeur du terrain. Les taux d'intérêt des prêts sont aussi subventionnés à hauteur de 40 \%. II est clair que la loi joue en faveur de l'intensification des concentrations des équipements touristiques.

Aux termes de la Loi 3299/2004 en vigueur depuis 2005, le pays est divisé de nouveau en trois zones d'incitation. Les subventions oscillent entre $15 \%$ et $35 \%$ du coût total de l'investissement hôtelier, y compris la valeur du terrain. Pour la première fois, les incitations concernant les régions déjà développées, comme Rhodes, sont d'intensité moyenne, cela après l'intervention de l'Union des hôteliers locale, qui reconnaît les problèmes de suréquipement de l'île et de fortes concentrations d'équipements hôteliers.

\section{Le financement bancaire du tourisme}

Depuis les années 1950 et jusqu'en 1997 l'économie grecque en est une d'endettement : le financement monétaire est soutenu par la Banque de Grèce et porte sur les crédits bancaires. Les pouvoirs publics, par la tutelle exercée sur les institutions financières, orientent l'allocation des ressources vers les secteurs jugés prioritaires et administrent les taux d'intérêt, qui sont maintenus artificiellement faibles ou même négatifs en valeur réelle.

Au cœur du système de financement bancaire se trouve la Banque de Grèce, contrôlée par l'État. II en résulte que la Banque n'est pas une institution indépendante, mais un moyen d'exercer la politique financière et monétaire du gouvernement, qui nomme son gouverneur. La Banque fixe les taux d'intérêt, surveille les transactions de devises et finance en grande partie les déficits budgétaires de l'État (CPRE, 1991 : 24). Au sein de la Banque de Grèce, la Commission monétaire constitue une structure particulièrement puissante, laquelle décide en réalité de la politique que la Banque doit mettre en œuvre. Les décisions de la Commission sont plus politiques que strictement économiques et financières. L'industrie et le commerce extérieur sont toujours les activités les plus privilégiées et leur financement a toujours été assuré (Chalikias, 1976), tandis que des crédits faciles à destination de l'hôtellerie ne sont accordés que depuis la fin des années 1960.

Depuis les années 1970, les dépôts bancaires privés sont très élevés. Jusqu'en 1990, les taux d'intérêt réels des comptes d'épargne sont considérablement inférieurs par rapport à l'indice général des prix (ill. 4), ce qui résulte en une perte importante de revenus pour les épargnants (Hondroyiannis, 2002). Évidemment, cela profite aux emprunteurs, dont le plus important est l'État. Malgré les faibles taux d'intérêt, l'épargne privée a toujours été élevée, ce qui crée une grande disponibilité de capitaux liquides. L'État récupère ces liquidités par le biais du mécanisme de contrôle bancaire précédemment décrit et il est ainsi en mesure de financer par crédits bancaires les activités considérées comme productives, par des capitaux bon marché (CPRE, 1991 : 18). Depuis 1973 et jusqu'au début des années 1990, les tensions inflationnistes sont de plus en plus préoccupantes. Afin de combler ses déficits et d'assurer le financement du développement économique, l'État a recours à la création monétaire. Pendant toute cette période, la liquidité des capitaux est maintenue à un niveau élevé (Panagiotopoulos, 1984 : 83 ; CPRE, 1991 : 247). La libéralisation du système bancaire (Loi 2076/1992) et le développement du marché boursier surviennent dans les années 1990. Pourtant, le marché boursier n'a jamais été une source de capitaux ; en 2007, seulement quatre entreprises hôtelières sont socialisées et cotées en bourse. Depuis 1997 (Loi 2548/1997), la Banque de Grèce est indépendante de l'État. Désormais sa mission est de veiller à la stabilité des prix et au contrôle des portefeuilles, des placements et de la crédibilité des banques.

La caractéristique marquante de tous les organismes et banques qui ont assuré le financement à long terme pour la création d'entreprises est qu'ils demeurent sous le 


\section{Illustration 4}

Évolution des taux d'intérêt des prêts bancaires à long terme, des taux d'épargne (simples) et de l'indice général des prix en Grèce (1960-2005)

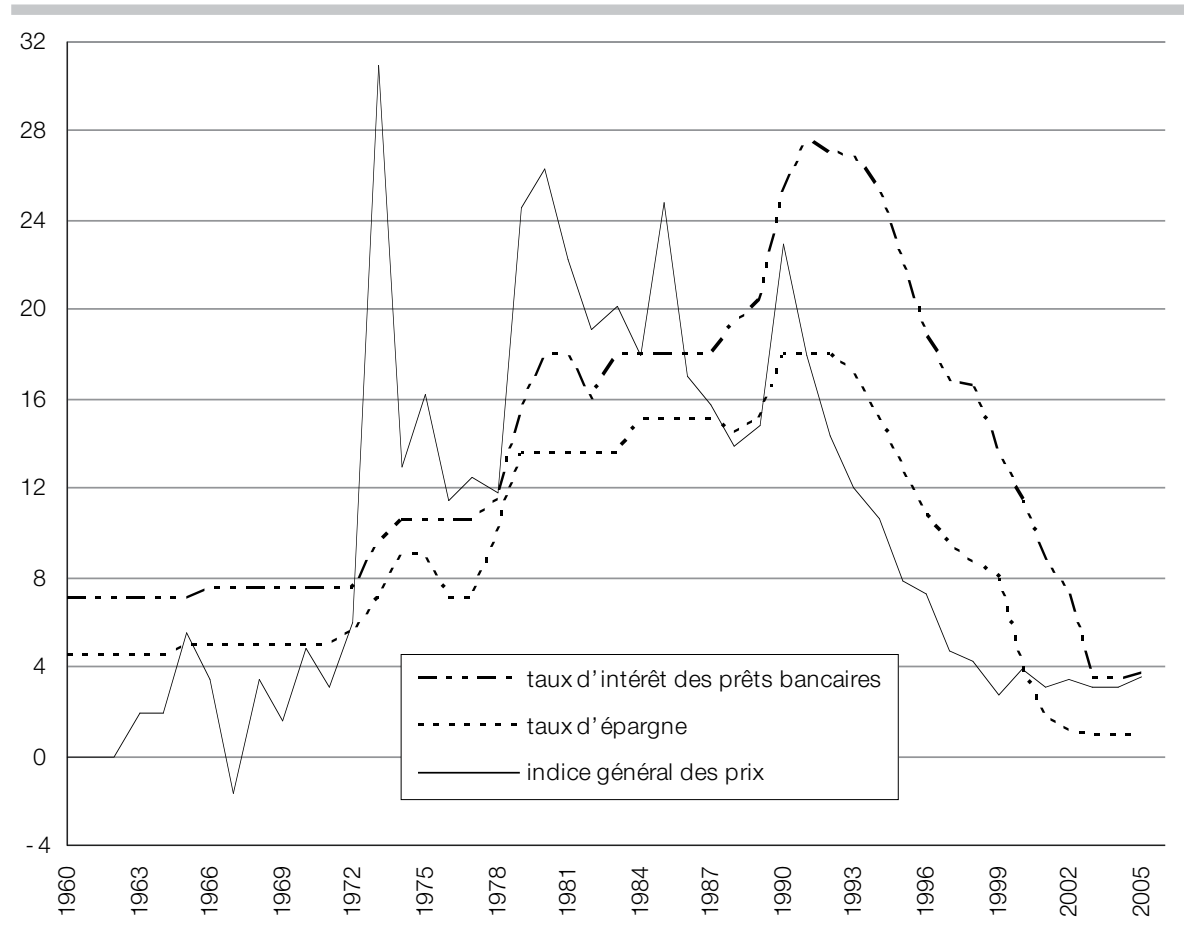

Source : Banque de Grèce.

contrôle de l'État. Jusqu'en 1963, l'Organisme de financement du développement économique, créé en 1954, et l'Organisme de crédit touristique sont chargés du financement du tourisme (Banque de Grèce, 1967 : 62). Ces deux organismes de financement sont incorporés à la Banque hellénique de développement industriel (BHDI), créée en 1964. Les autres établissements publics qui ont largement financé le secteur sont la Banque du crédit foncier et la Caisse des dépôts et consignations (BHDI, 1976 : 24).

Jusqu'au milieu des années 1990, les banques commerciales sont mobilisées dans le financement à court terme du tourisme (CPRE, 1991 : 25). Contrairement aux organismes publics, elles subissent le contrôle de l'État. Grâce à son autorité accordée par la loi, la Banque de Grèce est à même de contrôler directement la politique de constitution et de gestion des portefeuilles des banques commerciales du pays. Toutes sont obligées de déposer leurs liquidités sur un compte de dépôt à la Banque de Grèce. Ainsi, elles se trouvent dans une situation permanente de dépendance vis-à-vis de la Banque, qui, d'une part, contrôle leur extension financière et, d'autre part, canalise le financement des entreprises et des activités économiques vers les secteurs que la politique gouvernementale favorise. Avec l'objectif de maitriser le système financier, la Commission monétaire instaure d'innombrables réglementations, qui, en pratique, rendent impossible le contrôle des financements bancaires et entravent leur fonctionnement concurrentiel.

\section{Les rapports intimes entre les prix fonciers et le système de financement bancaire du tourisme : étude de cas}

Les effets des politiques de développement régional du tourisme sont d'importance variable selon l'organisation donnée du système de financement du tourisme. À la suite de subventions aux entreprises et de crédits bancaires facilement accordés par des banques publiques, c'est le phénomène de la hausse des valeurs foncières qui prend de l'ampleur dans les espaces touristiques. Les mécanismes du système de financement du secteur sont profondément dépendants des biens fonciers et immobiliers.

Pour l'analyse de l'impact des politiques de développement régional sur le marché foncier local, nous avons choisi comme terrain d'étude l'agglomération touristique littorale de Faliraki, sur l'île de Rhodes en Grèce (ill. 5).

L'île de Rhodes est située dans le sud de la mer Égée. En 1961, sa population s'élevait à 64381 et, selon le recensement de 2001, elle avait atteint 124000 habitants. Le développement touristique de l'île a été véritablement amorcé dans les années 1950. Depuis les années 1970, la progression des arrivées touristiques a été constante et spectaculaire. C'est ainsi que, depuis les années 1980, l'île est considérée comme une destination de tourisme " de masse ". Selon les statistiques officielles, les arrivées par voie aérienne de touristes internationaux ont dépassé les 1,3 million en 2003. En 2004, l'île comptait 522 hôtels pour une capacité excédant 75000 lits. En 2005, l'agglomération de Faliraki comptait pour sa part 75 hôtels pour une capacité de plus de 13000 lits, tandis que les équipements annexes représentaient environ 9000 lits, selon les estimations de la commune de Kalythies, à laquelle Faliraki appartient.

Contrairement au reste du pays, l'île de Rhodes a hérité son cadastre des colons italiens du début du siècle ; nous avons donc pu entreprendre une recherche dans les archives cadastrales ; celles-ci comprennent des documents qui permettent de localiser les parcelles et de connaître leurs différents propriétaires, mais aussi la situation juridique des biens et, surtout, quelles hypothèques les grèvent et au profit de qui. Le cadastre constitue donc une source de données absolument crédible et inestimable.

Dans la mesure où l'État garantit l'action entrepreneuriale par des lois de développement régional, les banques soutiennent la réalisation de projets d'investissements hôteliers. Outre les subventions étatiques, les fonds qui supportent l'hôtellerie, aussi bien à Rhodes que 
Illustration 5

\section{Évolution de la capacité hôtelière sur l'île de Rhodes entre 1980 et 2004}

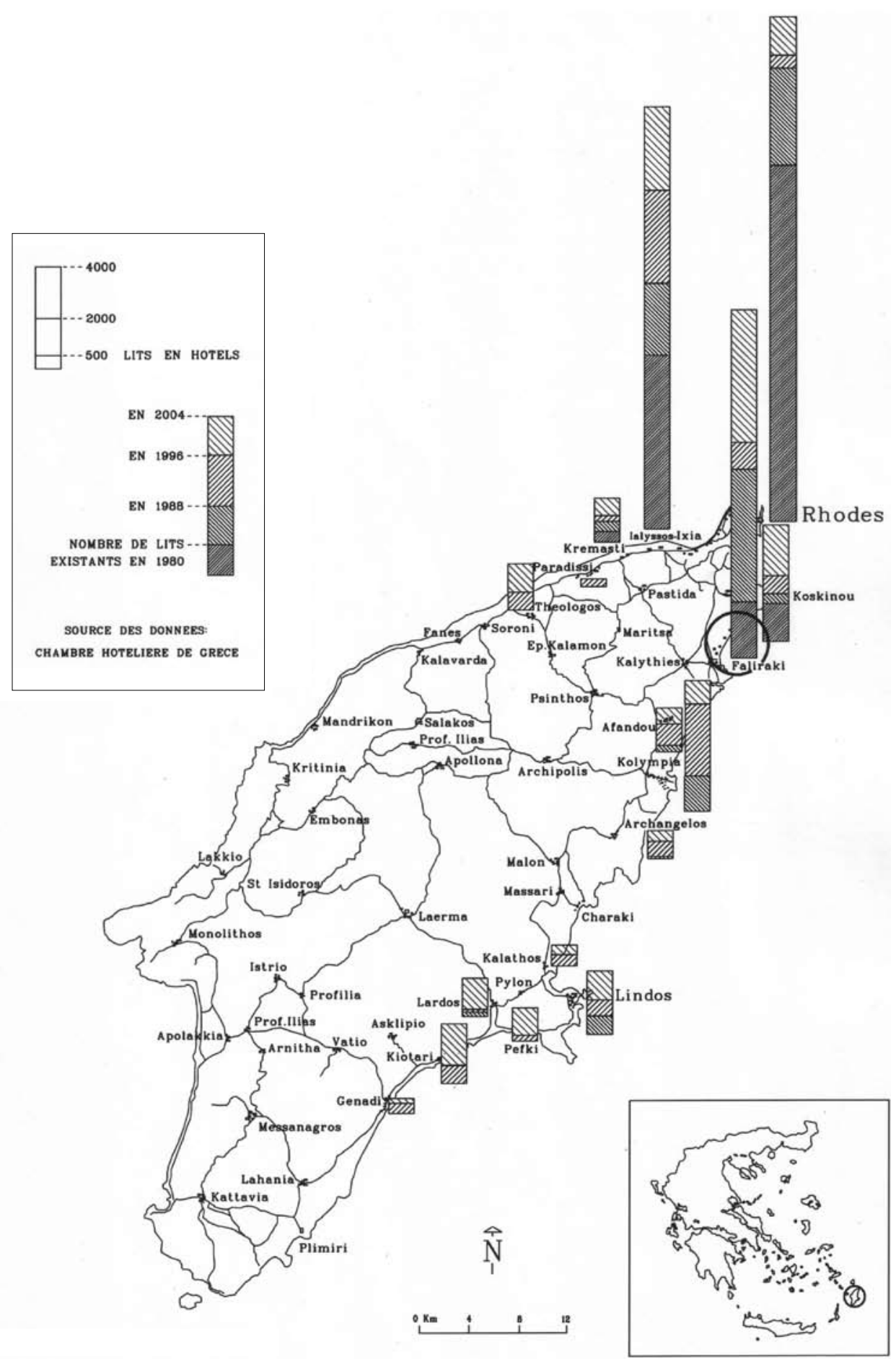

Source : auteur dans l'ensemble du pays, émanent d'un petit nombre d'établissements bancaires. Notre enquête sur les hypothèques concernant les entreprises inscrites dans le livre cadastral confirme le fait que la Banque hellénique de développement industriel (BHDI) a financé à plus de 80 \% les investissements hôteliers par des crédits à long terme, jusqu'aux quelques années précédant son rachat par la Banque du Pirée (Piraeus Bank) en 2002.

En règle générale et jusqu'à la fin des années 1980, il est particulièrement facile pour une entreprise d'obtenir un prêt. Paradoxalement, bien que la Commission monétaire ait toujours essayé d'exercer un contrôle strict sur la gestion des portefeuilles des banques commerciales privées, par le biais d'un système complexe de réglementations, elle accorde une pleine liberté d'action aux institutions publiques de financement (BHDI, 1976 : 64) et elle ne définit jamais les lignes directrices de leurs activités (Chalikias, 1976 : 214). C'est ainsi que leurs décisions de financement deviennent beaucoup plus sensibles aux pressions politiques et que les relations personnelles « préférentielles » entre leurs dirigeants et leurs clients sont favorisées. Dans un contexte de disponibilité de capitaux, le financement d'entreprises qui n'apportent pas toutes les garanties formellement exigées n'est pas une tâche difficile.

Les biens fonciers et immobiliers constituent les garanties réelles exigées pour l'obtention de prêts. Le dynamisme des entreprises ou leurs perspectives de rentabilité ne sont pas pris en compte (Vranopoulos, dans Karatzas, 1981 : 180). En réalité, même le critère bancaire des garanties réelles n'est pas respecté, étant donné que le fonctionnement des entreprises très endettées est continuellement financé. Dans certains cas, bien que la banque ait déclaré la confiscation et la vente aux enchères des biens d'entreprises, quelques mois plus tard, après avoir évité la confiscation, ces mêmes entreprises obtiennent des prêts importants de la même banque. Le fait que les biens des entreprises soient grevés d'hypothèques excédant largement leur valeur ne joue pas résolument sur la décision de financement. II est donc impossible d'arriver à des résultats quantifiables par l'analyse des données sur les hypothèques inscrites au livre cadastral. Très souvent elles sont 
particulièrement nombreuses et il est impossible de connaître l'état réel d'endettement des entreprises. La décision d'allocation du crédit est prise au siège central de la BHDI à Athènes, après examen du dossier constitué par son établissement départemental ou régional. L'enquête sur la situation juridique du bien hypothéqué est réalisée par le conseiller juridique de la banque, auprès du Bureau de conservation des hypothèques auquel appartient le bien, en l'occurrence le cadastre.

Cette politique déraisonnable du financement bancaire des entreprises résulte en l'apparition d'entreprises surendettées, dans un environnement entrepreneurial malsain. Ces entreprises mort-nées ont besoin d'une alimentation continue en capitaux, mais leur survie est de moins en moins garantie. Les établissements créditeurs se trouvent donc fatalement devant un choix difficile : soit continuer à les financer, soit procéder à la saisie de leurs biens. Dans le premier cas, les banques ont peu d'espoir d'être remboursées. Dans le deuxième cas, elles vont faire face à d'autres difficultés : d'une part, les banques ne peuvent pas faire fonctionner l'entreprise et, d'autre part, la vente aux enchères des biens n'est pas facile et, le cas échéant, ne leur rapporterait qu'une partie des capitaux empruntés. Ainsi, la « solution » la plus courante est de continuer à financer l'entreprise. Les intermédiaires politiques jouent souvent leur rôle de catalyseur. Puisque les entreprises en difficulté sont nombreuses, les pressions politiques sont concertées, exercées par les députés ou les ministres régionaux, par " devoir » pour leurs électeurs. Ainsi, ce système de financement par les banques publiques assure pour longtemps la continuité du fonctionnement des entreprises. Même après la privatisation des banques publiques comme la BHDI, les problèmes hérités de l'endettement excessif des entreprises persistent.

\section{L'effet multiplicateur des subven- tions pour la création d'entreprises sur les valeurs foncières \\ Les aides et surtout les subventions aux entre-} prises entraînent des effets multiplicateurs sur les prix fonciers. À noter que les valeurs foncières dans le marché local sont fixées suivant leur usage le plus rentable ; cela détermine aussi la qualité (la catégorie) et le plus souvent la taille des entreprises hôtelières.

Illustration 6

L'effet du taux de subvention sur les valeurs foncières

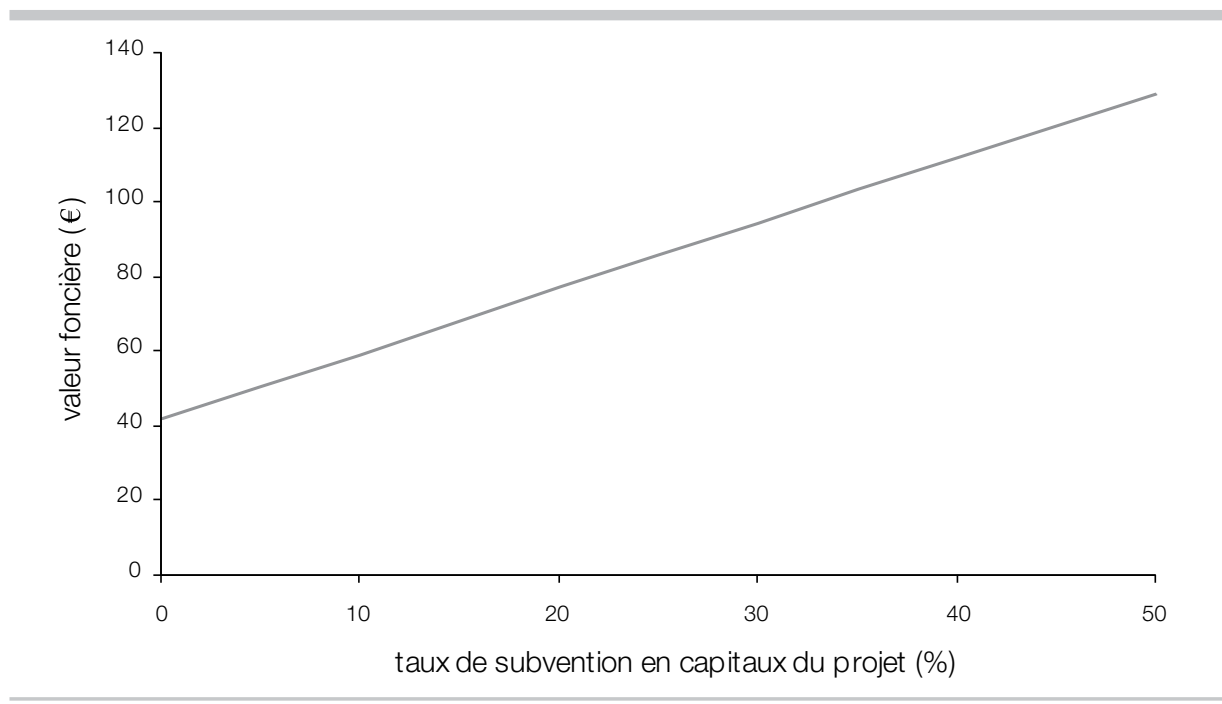

Source : Triantafyllopoulos, 1999.

Dans son œuvre majeure, Principes d'économie politique, publiée en 1890, Alfred Marshall (1890, livre V) développe une synthèse devenue classique de la théorie de la rente foncière. Selon lui, la rente foncière est un élément composite de plusieurs rentes moindres : rente foncière proprement dite qui dépend de la qualité du sol, rente de situation qui dépend des économies externes et quasi-rente liée aux instruments de production. Les économies externes ne résultent pas d'une dépense spéciale de la part de ceux qui en bénéficient, mais elles sont liées à l'environnement et à l'influence du milieu sur les coûts de production d'une entreprise. En définitive, pour Marshall, la rente (ou surplus) est un revenu ex post, résultant du profit de l'utilisation de la terre, après déduction des dépenses nécessaires à la production, tandis que les effets externes sont internalisés dans les prix fonciers.

Cette idée est intrinsèque dans le raisonnement du " compte à rebours ", selon lequel c'est le rendement du produit final (l'hôtel) qui détermine le prix du terrain. Grâce à l'utilisation des techniques d'évaluation des projets immobiliers d'investissements hôteliers, et plus particulièrement de la méthode du discounted cash flow (DCF) (PFK Consulting, 1996 ; Mitchell et Ingram, 2002 ; Nilsson et al., 2002 ; Fraser, 2004), il est possible de quantifier l'effet des subventions en capitaux sur les valeurs foncières dans l'espace d'étude. L'avantage de cette méthode est l'internalisation au sein du modèle aussi bien des composantes des rentes de situation que celles des quasi-rentes, par l'usage de données quantitatives.

Suivant la logique marshallienne, les coûts et les profits de l'instrument de production (les hôtels) sont :

- Le coût moyen de construction d'un hôtel. II s'agit du coût moyen par catégorie d'hôtel, calculé par l'Office national du tourisme grec tous les deux ans. C'est le même coût qui est utilisé par le ministère de l'Économie pour calculer le montant des investissements hôteliers qui seront subventionnés.

- Le prix moyen de location des chambres hôtelières par catégorie d'hôtels dans la région, disponible auprès de la Chambre hôtelière de Grèce. ॥ représente une moyenne des qualités du site et des hôtels qui s'y situent.

- Les autres recettes d'une entreprise de ce type (restauration, locations diverses, autres services annexes), ainsi que le coût de leur approvisionnement, tels qu'observés dans les entreprises hôtelières locales de la même catégorie. 
Les effets (économies) externes qui sont internalisés par l'instrument de production et dépendent du développement général de la région où se situe l'hôtel sont :

- Les taux d'occupation par catégorie d'hôtel dans l'agglomération d'étude, disponibles auprès de l'Office national de statistiques, à l'échelon départemental. Ces taux expriment l'intégration de l'hôtellerie locale dans le marché touristique, le niveau des infrastructures générales et celui des autres services qui soutiennent l'hôtellerie et le développement touristique.

- Les taux d'intérêt des prêts bancaires à long terme et le taux d'inflation, officiellement connus.

- Les effets des subventions et des autres aides de l'État au développement du secteur hôtelier dans la région, étant donné que le tourisme est l'activité dominante dans l'agglomération et que l'hôtellerie locale est subventionnée depuis très longtemps.

- Le risque de l'investissement, tel qu'il est appréhendé par les banques créditrices.

Dans notre cas, étant donné les valeurs foncières enregistrées dans l'espace d'étude, I'utilisation du modèle permet de calculer leur hauteur dans le cas où les subventions à la création d'entreprise n'existeraient pas. Ainsi, selon notre modèle, et pour une entreprise hôtelière fictive de catégorie quatre étoiles d'une capacité de 700 lits, située dans notre espace d'étude, des subventions à hauteur de $10 \%$ ou de $30 \%$ du coût total de l'investissement sont respectivement responsables de hausses de la valeur du terrain de l'ordre de $45 \%$ ou de $90 \%$ (ill. 6).

On ne peut donc que constater l'effet multiplicateur - particulièrement important - des subventions pour la création d'entreprises sur les valeurs foncières.

En supposant qu'il y ait un rapport entre les prix des chambres et ceux des terrains, l'écart entre les deux séries de données indique la dissociation croissante entre les valeurs d'usage et les prix fonciers. L'illustration 7 montre l'évolution

\section{IIlustration 7}

\section{Rythmes d'évolution des prix fonciers et des prix des chambres hôtelières dans l'espace d'étude}

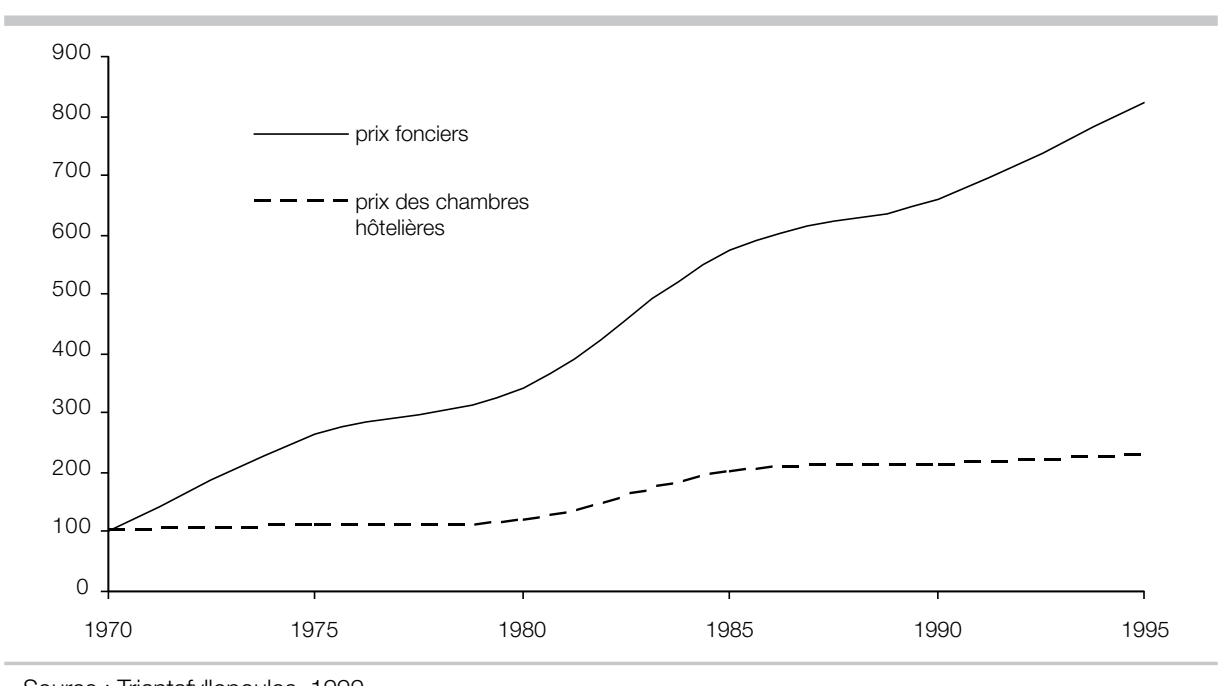

Source : Triantafyllopoulos, 1999.

comparative des prix constants du sol et des chambres hôtelières dans notre espace d'étude. Les prix d'acquisition du sol semblent devenir indépendants par rapport à l'usage et à la rentabilité économique que I'on peut en tirer. La production immobilière du tourisme suit un cercle vicieux. On se réfère de moins en moins aux "valeurs d'usage » des équipements hôteliers.

\section{Les effets de transfert de la rente foncière}

Le système de financement des activités touristiques est profondément marqué par l'interventionnisme étatique, aussi bien par des aides au développement régional que par le biais du contrôle du système de financement bancaire du secteur. Cependant, la territorialisation de cette politique suivie se concrétise et entraîne des impacts socioéconomiques et spatiaux complémentaires à l'échelle locale, conditionnés aussi bien par la mobilisation des acteurs locaux que par les politiques spatiales de l'État.

L'augmentation des prix fonciers apparaît comme un transfert de richesse entre les propriétaires des terrains et les entrepreneurs. Une transaction atteint le prix élevé qui est le sien dans la mesure où le vendeur cède des droits d'usage pour un avenir qui sera plus fructueux que le présent. Mais c'est un pouvoir d'achat actuel qui est versé pour acquérir le terrain : celui qui vend monnaye ses droits sur l'avenir en amputant les avoirs présents d'un acheteur éventuel. La hausse des prix fonciers modifie la répartition des richesses au profit des propriétaires des terrains.

On pourrait soutenir que ce transfert de richesses se fait au détriment des moyens de production, qui créent des richesses dans le présent, ce qui est une hypothèse juste et logique, à condition que la concurrence intersectorielle du capital touristique entre l'occupation du sol et la mise en place des moyens de production soit parfaite. Pourtant, il faut tenir compte des éléments suivants : a) les aides élevées de l'État à la création d'entreprises à forte intensité capitalistique, par des subventions et des prêts bancaires à conditions préférentielles et b) la valeur vénale élevée du sol, donc du paiement d'un surplus de la rente par les entreprises aux propriétaires des terrains. La propriété terrienne est très diffuse au sein des populations locales à Rhodes (Finas, 1991 : 55). La rente prélevée par les petits propriétaires fonciers locaux leur donne alors la possibilité de se mobiliser euxmêmes dans le secteur du tourisme et de s'y investir, selon les disponibilités de chacun ; ce qui se fait évidemment non seulement lors de la vente des terrains, mais également par leur exploitation touristique et par une multitude d'autres manières. Dans notre espace d'étude, le transfert de la rente se fait ainsi au 
profit des sociétés locales, qui s'investissent ensuite elles-mêmes dans le tourisme. Ce mécanisme de "socialisation " de la rente favorise alors la territorialisation du tourisme, autrement dit l'intégration des activités touristiques dans les sociétés locales, qui sinon seraient marginalisées, absentes du circuit économique et du processus de distribution des richesses qu'engendre le tourisme en tant que phénomène économique. Cette intégration constitue un fait particulièrement positif pour les sociétés locales.

\section{Conclusion}

Les politiques de financement du tourisme de l'État grec ont joué un rôle prépondérant, tant sur l'aspect économique que spatial du développement régional du tourisme. Pourtant, en l'absence de plans concrets définissant des objectifs socioéconomiques et spatiaux aux niveaux national et régional et des stratégies pour les atteindre, et en l'absence de politique d'aménagement, c'est le système de financement du tourisme qui a conditionné la distribution spatiale des équipements touristiques dans le pays, en fonction de facteurs locaux.

Les politiques de financement du développement régional se sont avérées favorables pour les moyennes entreprises touristiques, qui rencontrent très souvent des difficultés à obtenir des crédits. Néanmoins, elles sont à l'origine d'initiatives entrepreneuriales de la part de propriétaires fonciers qui n'avaient aucun rapport antérieur avec le tourisme, mais qui étaient simplement propriétaires de terrains de valeur importante, ainsi que de la création de nombreuses entreprises mort-nées ; le taux de participation des entrepreneurs au coût total des entreprises étant souvent particulièrement bas, la valeur des terrains y a été incluse. Un grand nombre d'entreprises ont été créées dans des régions où les conditions n'étaient pas propices au développement du tourisme : soit elles n'étaient pas dotées d'importantes ressources touristiques (absence de qualités naturelles et d'attractions, courte saison), soit les infrastructures nécessaires manquaient (Mylonas, 1996a et 1996b).

Une grande accessibilité aux crédits fonciers et immobiliers ainsi que les subventions de l'État augmentent le nombre de ceux qui peuvent participer au jeu de la hausse des prix fonciers. À côté de gens qui investissent leurs capitaux apparaissent d'autres qui interviennent sans apports personnels suffisants. L'opportunité du gain facile modifie aussi le comportement des propriétaires-investisseurs et les amène à se préoccuper non de l'opportunité et de la viabilité d'un investissement, mais des possibilités de tirer profit de leur propriété, dans les espaces « touristifiés » du pays, en raison du mode d'allocation des subventions étatiques. L'élévation des moyens financiers mis à la disposition des entreprises acheteuses est corrélative à l'augmentation des exigences des vendeurs de terrains : aussi bien les entreprises que les vendeurs ont donc intérêt à voir le prix du sol augmenter. Puisqu'aucune précaution ne vient interdire l'accroissement de la demande de capitaux et qu'aucun mécanisme de contrôle de l'espace et des prix fonciers ne subsiste, le foncier devient la base de la création de richesses.

Les aides aux entreprises et, surtout, les subventions accordées avec plus ou moins la même intensité sur les mêmes espaces pendant de longues périodes, ont favorisé la création de concentrations spatiales et créé les conditions d'un " effet de serre " dans certains espaces, c'est-à-dire un environnement entrepreneurial conditionné, sans rapport direct avec les évolutions de la demande touristique et les exigences locales. C'est ainsi qu'elles ont renforcé la concentration des investissements et le suréquipement de sites déjà développés et ce, pour deux raisons : d'abord parce que la valeur des terrains y était très élevée et que la contribution des entrepreneurs en capitaux propres était de ce fait moins importante ; ensuite parce que ces destinations touristiques étaient généralement déjà bien placées sur le marché du tourisme international et, par conséquent, la viabilité des investissements y était moins risquée. Or, la surproduction de l'offre met en position avantageuse les voyagistes puissants, qui exercent des pressions sur les hôteliers pour obtenir une baisse des prix. La faible rentabilité des entreprises est à l'origine de leur endettement et entraîne bien souvent vers le bas la qualité des services (Loukissas et Triantafyllopoulos, 1997), fait dénoncé à plusieurs reprises par la Chambre hôtelière de Grèce.
L'État a assuré des capitaux abondants et bon marché aux entreprises touristiques, surtout dans le passé, grâce à des lois relatives au développement régional et aux établissements créditeurs publics. Le rôle du foncier dans le système de financement des entreprises a toujours été essentiel. Par le biais de ce système, l'État facilite le transfert de capitaux, pas forcément vers les régions les moins développées en matière de création d'entreprises hôtelières, mais, en même temps, il valorise la propriété foncière locale qui devient le pivot de l'investissement privé du développement touristique local. La multiplication des valeurs foncières, due à une demande accrue de terrains pour des investissements hôteliers, favorise la création d'un capital local et la participation active de la société locale au processus de développement touristique, lui attribuant ainsi des caractéristiques de développement endogène.

Nikolaos Triantafyllopoulos est enseignant au Département d'aménagement et de développement régional de l'Université de Thessalie, Grèce.

\section{Bibliographie}

Armstrong, Harvey et Jim Taylor (2000), Regional Economics and Policy, Oxford, Blackwell Publishing.

Artobolevskiy, Sergey (1997), Regional Policy in Europe, Regional Policy and Development Series 11, London, Regional Science Association.

Banque de Grèce, Institut de Recherches Bancaires (1967) Le financement du tourisme. Athènes, Banque de Grèce, étude no 11 (en grec).

Banque de Grèce (1996), Rapport du gouverneur pour l'année 1995, Athènes, Banque de Grèce.

Banque hellénique de développement industriel (BHDI) (1976), Recherche en tourisme, Direction de recherches, Athènes (en grec).

Bar-On, Raphael Raymond et Moshe Even-Zahev (1995), Investments and Financing in the Tourism Industry, Jerusalem, Israel Ministry of Tourism.

Capello, Roberta (2007), Regional Economics, New York, Routledge.

Cazes, Georges (1992), Fondements pour une géographie du tourisme et des loisirs, Paris, Bréal, coll. "Amphi géographie ».

Centre de planification et de recherches économiques (CPRE) (1976), Rapport pour le Plan 1976-1980 : Tourisme (rapport n 6), Athènes (en grec).

Centre de planification et de recherches économiques (CPRE) (1987), Tourisme. Rapport pour le Plan 1983-1987, Athènes (en grec). 
Centre de planification et de recherches économiques (CPRE) (1991), Programme de développement régional. Incitations pour le développement touristique, Rapport du département du Tourisme, Athènes (en grec).

Chalikias, Dimitris (1976), Possibilités et problèmes de la politique de crédit, Athènes, Banque de Grèce (en grec).

Cooper, Chris, Stephen Wanhill, John Fletcher, David Gillbert et Alan Fyall (1998 [2e éd.]), Tourism. Principles and Practice, Essex, Longman.

Ivars-Baidal, Joseph (2004), "Regional Tourism Planning in Spain. Evolution and Perspectives", Annals of Tourism Research, vol. 31, no 2 , p. 313-333.

Finas, Kyriakos (1991), L'économie du Dodécanèse depuis son rattachement à la Grèce jusqu'à nos jours, 1947-1989, Rhodes, Chambre de commerce et d'Industrie du Dodécanèse (en grec)

Fraser, W.D. (2004), Cash-flow Appraisal for Property Investment, New York, Palgrave Macmillan.

Göymen, Korel (2000), « Tourism and Governance in Turkey ", Annals of Tourism Research, vol. 27 , n 4 , p. 1025-1048

Hondroyiannis, Giorgos (2002), « Dépôts privés en Grèce : facteurs déterminants, économiques et démographiques ", Bulletin économique, $n^{\circ} 19$, Athènes, Banque de Grèce (en grec).

Igoumenakis, Nikos (1997), Politique touristique, Athènes, Editions Kritiki (en grec).

loannides, Dimitri et Keith G. Debagge (dir.) (1997), The Economic Geography of the Tourist Industry. A Supply-side Analysis, New York, Routledge.
Karatzas, Konstantinos (dir.), (1981) Résumés des rapports de la commission dirigée par $N$. Charissopoulos pour l'étude du système bancaire grec, Athènes, Banque de Grèce (en grec).

Loukissas Philippos et Nikolaos Triantafyllopoulos (1997), Factores de competitividad para destinos turisticos tradicionales, Papers de Turisme, no 22, p. 115-129.

Marshall, Alfred (1890), Principles of Economics, [http://www.econlib.org/library], consulté en février 2008

Mitchell, P. et H. Ingram (2002), " Space Revenue and Valuation Models in Retailing and Hotels ", International Journal of Contemporary Hospitality Management, vol. 14, $\mathrm{n}^{\circ} 1$, p. 28-33.

Montanari, Armando et Allan Williams (dir.) (1995), European Tourism. Regions, Spaces and Restructuring, Etobicoke (Ont.), Wiley.

Mylonas, Annivas (1996a), Tourisme: Politique, évolution, perspectives et contribution à l'économie, Rapport interne du Centre de planification et de recherches économiques, Athènes (en grec).

Mylonas, Annivas (1996b), «Tourisme: Tendances et perspectives ", dans Andreas Kintis (dir.), Banque lonienne 2004 : L'économie grecque au seuil du XX/e siècle, Athènes, Banque lonienne (en grec).

Nilsson, M., P. Harris et R. Kett (2002), " Valuing Hotels as Business Entities ", Journal of Leisure Property, vol. 2, n 1, p. 17-28.

Panagiotopoulos, Dimitris (1984), La politique monétaire grecque. L'expérience des vingt dernières années, Athènes, édité par l'auteur (en grec).
Papadoulis, Apostolos, Giorgos Petrakos et Yiannis Psycharis (2004), "Politiques d'incitations à l'investissement et développement régional : revue et première évaluation ", Revue de sciences économiques, vol. 5, no 1 , p. 5-40 (en grec).

Pearce, Douglas et Richard Butler (dir.) (1999), Contemporary Issues in Tourism Development, New York, Routledge.

Petrakos, George et Yiannis Psycharis (2004), Le développement régional en Grèce, Athènes, Editions Ktiriki (en grec).

PFK Consulting (1996), Hotel Development, Washington (DC), Urban Land Institute.

Seaton A.V. (dir.) (1994), Tourism. The State of the Art, Sussex, Wiley

Sinclair M.T. et S.J. Page (1993), « The Euroregion: A New Framework for Tourism and Regional Development ", dans J. Mawson, Policy Review Section, Regional Studies, vol. 27, n० 5, p. 475-482.

Triantafyllopoulos, Nikolaos (1999), " Les mécanismes fonciers de l'urbanisation touristique du littoral en Grèce ", thèse de doctorat non publiée, Unité de formation et de recherche (UFR) de géographie, Université de Paris Panthéon-Sorbonne.

Vanhove, Nobert (1999), Regional Policy: A European Approach, Aldershot, Ashgate.

Weaver, David (dir.) (2001), The Encyclopedia of Tourism, Oxon, Cab International.

World Tourism Organization (WTO) (1994), National and Regional Tourism Planning, Athènes, International Thomson Business Press.

Wren, Colin (2005), "Regional Grants: Are They Worth It? ", Fiscal Studies, vol. 26, n 2, p. 245-275.

\section{TÉQROS \\ Revue de recherche en tourisme \\ En dehors des dossiers thématiques Téoros ouvre ses pages aux propositions spontanées. La rédaction invite tous les chercheurs qui oeuvrent dans le domaine du tourisme ou qui s'intéressent au tourisme sous tous ses aspects, à soumettre des articles de nature analytique à la revue. On peut soumettre un article en l'envoyant à :}

\section{Appel à textes}

\section{teoros@uqam.ca}

Publiée trois fois l'an depuis 1982, la revue Téoros de recherche en tourisme s'affirme dans la francophonie comme le leader de diffusion des résultats de recherche et de réflexions fondamentales et appliquées dans une perspective multidisciplinaire. Les problématiques liées aux rapports entre le tourisme et la culture ou entre le tourisme et la société, à la gestion ou à la planification en tourisme, à des cas particuliers de mise en tourisme ainsi qu'aux questions méthodologiques d'actualité, par exemple, comptent parmi les sujets abordés.

Les textes soumis doivent apporter une contribution scientifique originale, que ce soit par le biais d'information factuelle, jusqu'alors inconnue ou par une nouvelle interprétation d'un thème particulier. Téoros vise avant tout le transfert de connaissances; son objectif est donc de promouvoir une meilleure compréhension des phénomènes liés au tourisme.

Les auteurs doivent faire parvenir un manuscrit présenté selon les règles de la revue, disponibles au www.teoros.uqam.ca.

Habituellement, un article analytique compte environ 6000 mots et n'excède pas 8000 mots avec trois ou quatre illustrations. On pourra cependant considérer des textes plus longs ou plus courts. Les articles peuvent être soumis en anglais ou en français et doivent être accompagnés d'un résumé de 100 à 200 mots (français/anglais) et de cinq mots-clés.

La publication des articles se fait sous réserve d'une évaluation. Tous les manuscrits seront évalués anonymement par des pairs qui pourront faire des suggestions ou demander des modifications. La rédaction transmettra l'avis des évaluateurs aux auteurs et s'assurera que les modifications demandées seront apportées.

Au plaisir de vous lire dans nos pages. 